\title{
Determining the sub-Lyapunov exponent of delay systems from time series
}

\author{
Thomas Jüngling, ${ }^{*}$ Miguel C. Soriano, and Ingo Fischer \\ Institute for Cross-Disciplinary Physics and Complex Systems, IFISC (UIB-CSIC), Campus University of the Balearic Islands, \\ E-07122 Palma de Mallorca, Spain
}

(Received 18 December 2014; published 9 June 2015)

\begin{abstract}
For delay systems the sign of the sub-Lyapunov exponent (sub-LE) determines key dynamical properties. This includes the properties of strong and weak chaos and of consistency. Here we present a robust algorithm based on reconstruction of the local linearized equations of motion, which allows for calculating the sub-LE from time series. The algorithm is inspired by a method introduced by Pyragas for a nondelayed drive-response scheme [K. Pyragas, Phys. Rev. E 56, 5183 (1997)]. In the presented extension to delay systems, the delayed feedback takes over the role of the drive, whereas the response of the low-dimensional node leads to the sub-Lyapunov exponent. Our method is based on a low-dimensional representation of the delay system. We introduce the basic algorithm for a discrete scalar map, extend the concept to scalar continuous delay systems, and give an outlook to the case of a full vector-state system, from which only a scalar observable is recorded.
\end{abstract}

DOI: 10.1103/PhysRevE.91.062908

PACS number(s): 05.45.Pq, 02.30.Ks, 89.75.-k, 05.45.Tp

\section{INTRODUCTION}

The exponential divergence of nearby trajectories is one of the essential properties of chaotic dynamics. The average rate of divergence is measured by the Lyapunov exponents that form a spectrum characterizing the evolution of small volumes in phase space. They further have consequences for the dimensionality of the attractor and the entropy rate. The determination of Lyapunov exponents is mostly restricted to theoretical works because their calculation requires the reconstruction of the local neighborhood of a trajectory. Furthermore, it requires information about all degrees of freedom as well as negligible noise levels, which is why experimentally the determination of Lyapunov exponents from time series is a rarely addressed challenge. Given a trajectory of a dynamical system, basic methods to approach Lyapunov stability [1-5] use the ergodic property of a chaotic flow to return to an earlier visited point in phase space arbitrarily close in the long term of its dynamic evolution. Thus, it allows for a nearest-neighbor reconstruction of the local vector field, which can also be carried out in the embedding space [6], if only a scalar time series has been recorded.

However, the application of embedding algorithms to time series obtained from high-dimensional systems is not practical [7]. A phase space vector in such a system can easily consist of hundreds degrees of freedom, where the probability to find nearest neighbors in this space is negligible. Here we tackle this problem for particular high-dimensional systems by presenting a simple algorithm to extract information about the chaotic trajectories of delay systems [8].

In recent decades, delay systems have become the focus of a myriad of works in the field of nonlinear dynamics and complex systems [9-11]. The simplest representation of a delay system is a nonlinear dynamical node with timedelayed feedback such that the equations of motion read $\dot{\mathbf{x}}(t)=\mathbf{f}(\mathbf{x}(t), \mathbf{x}(t-\tau))$ with $\mathbf{x} \in \mathbb{R}^{d}$ and the delay time $\tau>0$. This general notion can also be extended to the case of a delay-coupled network of different nonlinear nodes where the

\footnotetext{
*thomas@ifisc.uib-csic.es
}

coupling delays stem from finite signal propagation velocities. Many variations in this class of systems have been studied, including those with multiple delays, variable delays, and delay distributions. One may distinguish delay systems by the type of nonlinear node and by the amount of delay. The phase space of delay systems formally has infinite dimensions. This becomes clear when the evolution a delay system needs to be determined: The entire state $\mathbf{x}(t)$ for $t \in[-\tau, 0]$ needs to be given as initial conditions. This high dimensionality favors, but not necessarily implies, a high-dimensional chaotic dynamics depending on system and parameters. Hyperchaos with hundreds of positive Lyapunov exponents has already been identified from numerical simulations [12-14].

Previous studies on time-series analysis of delay systems focused on their identification, reconstruction of the equations of motion, and estimating the delay time [15-17]. These works highlight the relevance of several aspects, such as discrete versus continuous time, scalar versus vector character of the system, and the relationship between the feedback variable and the recorded variable, for the implementations of the corresponding methods. A common core feature of the existing methods lies in the embedding of a time series. Instead of sampling the entire delay interval in order to approximate the infinite-dimensional state vector, sampling a limited number of points at the edges of the delay interval, i.e., around time $t$ and around time $t-\tau$, reveals sufficient information about the dynamics. This embedding is of clear practical advantage when dealing with large delays. In delay systems one often distinguishes between the small delay regime and the large delay regime, which is defined by a time-scale separation between the delay time and the characteristic time scales of the nondelayed part of the system [18]. While for small delays the same time-series analysis methods as for nondelayed systems (see, e.g., [5]) can be applied, for large delays a different approach will be necessary. In this paper we focus on the latter, but our approach is not principally restricted to large delays.

In optical systems, delays in feedback or coupling connections often occur generically, due to their fast characteristic time scales. Moreover, these systems are versatile and well controllable and are therefore particularly suitable for the study of delay dynamical systems. Prominent examples for the 
versatility of optical delay systems are the experimental and theoretical studies of semiconductor lasers subject to optical feedback and of optoelectronic oscillators, which have proven very fruitful $[19,20]$. In these systems, the delayed feedback may induce complex intensity and frequency fluctuations that originate in high-dimensional chaos. Comparing the time scales of the laser with the feedback delay, one often finds that the device is operating in the large delay regime [21]. Considering the delayed feedback as an external drive of the nonlinear node offers a promising perspective in this case $[22,23]$. Then the response of the node can be characterized by the sub-Lyapunov exponent (sub-LE). ${ }^{1}$ The sub-LE is tightly connected to the maximum Lyapunov exponent and determines its scaling behavior especially by variation of the delay. The sub-LE can be considered an essential quantity underlying the chaotic dynamics, defining properties such as strong and weak chaos [26]. In particular, a positive sub-LE defines the regime of strong chaos, which cannot be synchronized, while a negative sub-LE refers to weak chaos, which in principle can be synchronized. In addition, the sub-LE determines the consistency properties of the dynamical node with respect to the delayed feedback signals. The analogy to the drive-response scheme is limited in the sense that the sub-LE is not part of the full Lyapunov spectrum of the system, whereas in the original drive-response scheme the conditional exponent does appear [25]. For the case of semiconductor lasers subject to optical feedback, nontrivial transitions of the sign of the sub-LE have been documented by variations of feedback or pump parameters only, corresponding to transitions between strong and weak chaos [27-29].

Since the sub-LE is determined by the response of the nonlinear node only, we aim at an algorithm using a lowdimensional embedding in order to calculate this quantity from time series. Naturally, this leads to the already proposed methods of reconstructing the equations of motion locally. An important and comprehensive contribution is provided by the works of Bünner and co-workers [17,30,31]. These studies include the calculation of Lyapunov spectra for the entire delay system. Our study on the sub-LE complements these findings by applying and extending the existing techniques. Beyond this, the perspective of drive-response systems, which leads to the sub-LE, offers additional insights into the origin of the emerging delay dynamics that cannot be obtained by reconstruction of phase space or the equations of motion alone.

In Sec. II we introduce the basic algorithm by means of a scalar discrete map. The performance is demonstrated by time series of the logistic map with delayed feedback. In Sec. III we extend the concept to the case of a scalar continuous delay system and exemplify the application of the algorithm with the Ikeda delay system. We further apply the method to experimental time series from an optoelectronic system. Section IV deals with the extension to a vector-state system. By means

\footnotetext{
${ }^{1}$ In Ref. [24] the term sub-Lyapunov exponent was first used for the conditional Lyapunov exponent, where the latter was the typical notation for this type of exponent after Ref. [25]. We use the different notations to distinguish between the sub-LE of a delay system and the conditional Lyapunov exponent in a nondelayed drive-response scheme.
}

of numerical simulations of the Lang-Kobayashi equations for semiconductor lasers with delayed optical feedback, we show the performance of the algorithm in the case in which the vector state is known. We finally give an outlook to the case of a scalar time series of a vector system. In the case of the LangKobayashi trajectories, we apply the algorithm to intensity time traces. We introduce a multiple-delay embedding in order to guarantee a reasonable performance of the algorithm for the sub-LE. We conclude with a discussion on the limitations and possible extensions. Details of the implementation are presented in Appendixes A and B, addressing the performance of the method under variation of parameters as well as on time series with measurement noise.

\section{BASIC ALGORITHM}

We consider a scalar discrete map with delay $\tau \in \mathbb{N}$,

$$
x_{n+1}=M\left(x_{n}, x_{n-\tau}\right) \text {. }
$$

We denote the time-dependent derivatives with respect to the first and second arguments by $a_{n}=D_{1} M\left(x_{n}, x_{n-\tau}\right)$ and $b_{n}=$ $D_{2} M\left(x_{n}, x_{n-\tau}\right)$, respectively. The tangent linear system of the map describing an infinitesimal perturbation reads

$$
\delta x_{n+1}=a_{n} \delta x_{n}+b_{n} \delta x_{n-\tau}
$$

and the reduced tangent system incorporates only the nondelayed term

$$
\delta \xi_{n+1}=a_{n} \delta \xi_{n}
$$

Note that the latter is driven by the same sequence of coefficients $a_{n}$ as the complete linearization originating from the full delay system. Iteration of the set of equations (1)-(3) with typical initial conditions leads, after the decay of transients, to a stationary trajectory $x_{n}$, along with accompanying trajectories $\delta x_{n}$ and $\delta \xi_{n}$. From the average exponential evolution of $\delta x_{n}$, one obtains the maximum Lyapunov exponent $\lambda$ of the dynamics. Accordingly, from $\delta \xi_{n}$ one obtains the subLyapunov exponent $\lambda_{0}$, which by its sign determines the property of strong or weak chaos.

Reconstructing the local linearized map by the coefficients $a_{n}$ and $b_{n}$ will allow us to calculate the sub-LE $\lambda_{0}$, which is given by

$$
\lambda_{0}=\left\langle\ln \left|a_{n}\right|\right\rangle_{n}
$$

where $\langle\cdot\rangle_{n}$ denotes the average over $n=\{1, \ldots, N\}$ with $N$ being the length of the trajectory, from which, in the formal definition of the Lyapunov exponent, the limit $N \rightarrow \infty$ should be taken. Note that a reconstruction of the local linear map would in principle also allow us to calculate the entire Lyapunov spectrum of the whole delay system [31].

We first approximate the evolution of the infinitesimal perturbations in Eq. (2) by finite perturbations obtained from nearest neighbors. For each value of the trajectory $x_{n}$ we search for its nearest neighbors $x_{m_{n, j}}$ with $j \in\{1, \ldots, J\}$. The index $m_{n, j}$ is the position in discrete time of the $j$ th nearest neighbor of the pair $\left(x_{n}, x_{n-\tau}\right)$. We calculate for each $(n, j)$ the difference $\Delta x_{j, n}=x_{m_{n, j}}-x_{n}$ and its $\tau$-delayed part acting as small perturbations. The indices are defined such that we obtain an ordering according to the chosen norm $d_{j, n}=\left\|\left(\Delta x_{j, n}, \Delta x_{j, n-\tau}\right)\right\|$, meaning that $d_{j, n} \leqslant d_{j+1, n}$. For a 
description of the chosen norm, length of time series, and number of nearest neighbors see the Appendixes. From the obtained neighborhood of $\left(x_{n}, x_{n-\tau}\right)$ we approximate the local linear map by

$$
\Delta x_{j, n+1}=\tilde{a}_{n} \Delta x_{j, n}+\tilde{b}_{n} \Delta x_{j, n-\tau}+\epsilon_{j, n},
$$

where by linear regression with respect to $\tilde{a}$ and $\tilde{b}$ we minimize the total error $E_{n}^{2}=\sum_{j} \epsilon_{j, n}^{2}$. This type of error is referred to as the one-step forecast error as discussed in detail in Ref. [17]. In a noise-free system, for the limit $\Delta x \rightarrow 0$ one may expect the convergence $\tilde{a}_{n} \rightarrow a_{n}$ and $\tilde{b}_{n} \rightarrow b_{n}$.

Our suggested procedure is exemplified by the logistic map with delayed feedback

$$
x_{n+1}=(1-\kappa) L\left(x_{n}\right)+\kappa L\left(x_{n-\tau}\right),
$$

where $L(x)=4 x(1-x)$. The map is iterated starting from a $(\tau+1)$-component vector of uniform random numbers in the unit interval, serving as initial conditions of the delay system. After a transient of $10^{5}$ delay times we assume that the trajectory has reached the attractor, which for most values of $\kappa$ is found to be chaotic, and we use time series from this point on for the Lyapunov analysis. For the logistic map the coefficients simply read $a_{n}=(1-\kappa) 4\left(1-2 x_{n}\right)$ and the correct sub-LE can be calculated from the average according to Eq. (4).

We apply the described reconstruction method in order to approximate the $a_{n}$ from the time series only. From a time series of $N=10^{6}$ points a standard nearest-neighbor search routine selects the $J=400$ points serving to form the perturbations $\Delta x_{j, n}$ and their corresponding values at time $n-\tau$ and $n+1$. Linear regression for every time step based on Eq. (5) reveals the reconstructed local linear map and hence an approximation of the sub-LE. Figure 1 shows the sub-LE from the true and reconstructed linear map as a function of the feedback strength $\kappa$. The presented range includes different dynamical regimes, from which most are chaotic, including three transitions between strong and weak chaos. The reconstruction reveals a perfect result in the entire range.

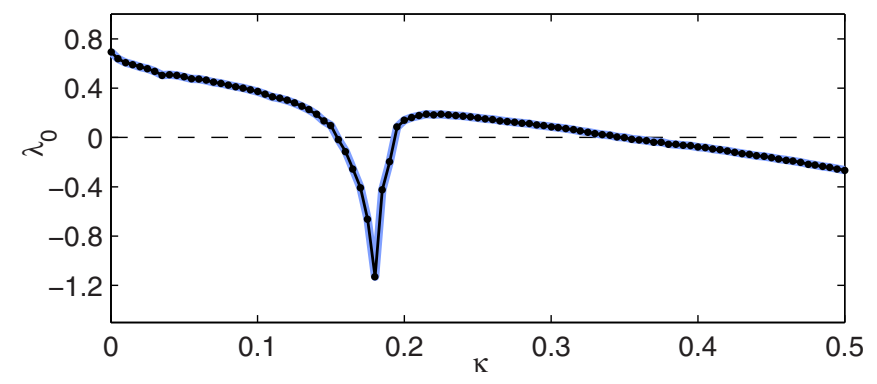

FIG. 1. (Color online) Sub-LE of the logistic map with delayed feedback $\tau=100$ as a function of the feedback strength $\kappa$. The blue (thick gray) line shows the true exponent from linearization and the narrow black line with markers shows the approximation to the sub-LE obtained from nearest-neighbor reconstructions perfectly coinciding with true sub-LE. There are three transitions between strong and weak chaos.

\section{CONTINUOUS SYSTEM}

A continuous system can be treated like a discrete map if data with sufficient accuracy and time resolution are available. However, for finite step sizes and resolution we find that the reconstruction of a useful local linear map requires a more sophisticated method in order to obtain reasonable results. We extend the algorithm introduced above, starting from the general case of a scalar continuous delay system with a single constant delay $\tau>0$,

$$
\dot{x}(t)=f(x(t), x(t-\tau)) .
$$

In analogy to the map we define the time-dependent linearizations $u(t)=D_{1} f(x(t), x(t-\tau))$ and $v(t)=D_{2} f(x(t)$, $x(t-\tau))$ by which we write the tangent linear systems for $\lambda$ and $\lambda_{0}$, respectively,

$$
\begin{aligned}
& \dot{\delta} x(t)=u(t) \delta x(t)+v(t) \delta x(t-\tau), \\
& \dot{\delta} \xi(t)=u(t) \delta \xi(t) .
\end{aligned}
$$

We aim to approximate the local linear system given by Eq. (6) from finite perturbations. For a finite time step $\delta t$ the integral version of this equation is obtained by the variation-of-constants formula

$$
\begin{aligned}
\delta x(t+\delta t)= & A(t+\delta t, t) \delta x(t) \\
& +\int_{t}^{t+\delta t} d t^{\prime} A\left(t+\delta t, t^{\prime}\right) v\left(t^{\prime}\right) \delta x\left(t^{\prime}-\tau\right)
\end{aligned}
$$

with the evolution operator of Eq. (7) given by

$$
A\left(t_{2}, t_{1}\right)=\exp \left\{\int_{t_{1}}^{t_{2}} u(t) d t\right\} .
$$

Discretizing the time to $t_{n}=n \delta t$ and $\delta x_{n}=\delta x\left(t_{n}\right)$, we can reduce the map (8) to

$$
\delta x_{n+1}=a_{n} \delta x_{n}+\int_{t_{n}}^{t_{n+1}} d t b(t) \delta x(t-\tau),
$$

with $a_{n}=A\left(t_{n+1}, t_{n}\right)$ and $b(t)=A\left(t_{n+1}, t\right) v(t)$ for $t \in\left[t_{n}, t_{n+1}\right)$. The integral term cannot be reduced further; accordingly, we have to deal with a functional expression. This functional is the linearization of the general expression described in Ref. [17]. Using $K$ support points $\theta_{k} \in[0, \delta t]$ to sample the delay term integral, we approximate the linear functional map. Nearest neighbors are determined in the space of the $(K+1)$ tuple $\left(x\left(t_{n}\right), x\left(t_{n}-\tau+\theta_{1}\right), \ldots, x\left(t_{n}-\tau+\theta_{K}\right)\right)$ to calculate the perturbations $\Delta x_{j}\left(t_{n}\right)$ and their $K$ delayed counterparts. The approximated local linear map reads

$$
\Delta x_{j}\left(t_{n+1}\right)=\tilde{a}_{n} \Delta x_{j}\left(t_{n}\right)+\sum_{k=1}^{K} \tilde{b}_{k} \Delta x_{j}\left(t_{n}-\tau+\theta_{k}\right)+\epsilon_{j, n} .
$$

From the regression coefficients $\tilde{a}_{n}$ we calculate $\tilde{\lambda}_{0}$ like for the discrete map. Compared to the discrete map in the previous section, in which only noise and nonlinear terms contribute to the error, in the continuous case the error of the integral approximation also contributes to the total error.

Employing the example of the Ikeda delay system, we demonstrate the method and address the issue of the 


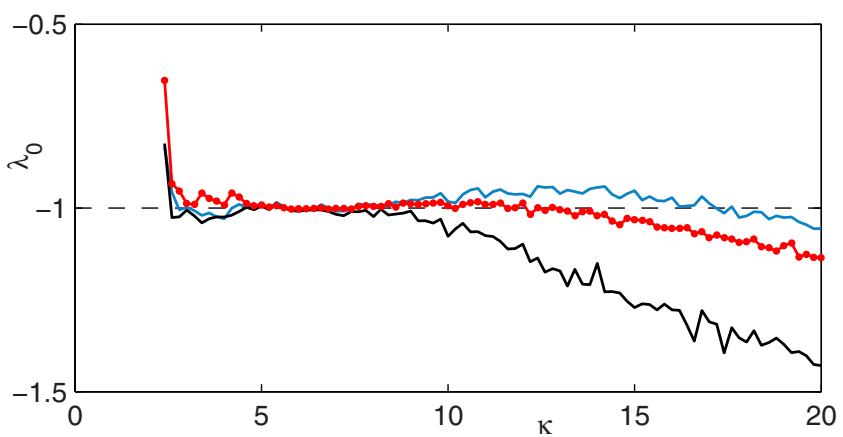

FIG. 2. (Color online) Sub-LE of the continuous Ikeda delay system for $\tau=10$. By construction $\lambda_{0}=-1$ for all $\kappa$ as indicated by the dashed black line. The reconstruction algorithm is applied with three different sampling methods: black, $\delta t=0.4$ and one sampling point at $\theta=0.2$; blue (gray), $\delta t=0.2$ and one sampling point at $\theta=0.1$; red (dark gray) with dots, $\delta t=0.4$ and two sampling points located at $\theta_{1}=0.1, \theta_{2}=0.3$. In the steady-state regime at $\kappa<2.3$, the reconstruction is not possible. At $2.3<\kappa<2.9$ the dynamics is periodic. The subsequent chaotic dynamics is characterized by amplitudes and bandwidth increasing with $\kappa$. At low feedback the sub-LE is recovered correctly even for the coarse sampling. With increasing feedback the reconstruction error increases as well, so a proper calculation of the sub-LE requires smaller $\delta t$ or higher $K$.

approximation of the integral. The reduced equations of motion read

$$
\dot{x}=-x+\kappa \sin \left(x_{\tau}\right)
$$

and its linearization is consequently

$$
\dot{\delta x}=-\delta x+\kappa \cos \left(x_{\tau}\right) \delta x_{\tau} .
$$

Due to the constant instantaneous term, it follows immediately that $\lambda_{0}=-1$ for all values of the feedback $\kappa$. Further, any reconstruction of the local linear map with a step $\delta t$ should reveal a constant coefficient $a_{n} \equiv \exp (-\delta t)$. We integrate Eq. (11) using a Heun method with step size $d t=0.001$ for various values of $\kappa$ and a delay time fixed at $\tau=10$, which can be considered a large delay. We discretize the numerical time series by a step size $\delta t$ and obtain the grid $t_{n}=n \delta t$. The nearest-neighbor reconstruction is applied correspondingly to the previous section. Figure 2 shows the obtained sub-LE from the reconstructed local linear maps compared with the true value for different step sizes $\delta t$ and different numbers of integral samplings $K$. By variation of $\kappa$ the system undergoes a bifurcation route starting at steady state for $\kappa=0$. For the steady state below $\kappa \approx 2.3$ the reconstruction fails due to missing nearest neighbors, while for the small periodic window between $\kappa \approx 2.3$ and $\kappa \approx 2.9$ the reconstructed map already reveals reasonable results. In the subsequent chaotic regimes the sub-LE is recovered well with a tendency of an increasing error for large feedbacks. The dynamics for low and high feedback differs in its amplitude and bandwidth, which both affect the performance of our algorithm. By the amplitudes increasing proportional to $\kappa$, according to the equations of motion, an increasing number of oscillations of the nonlinear delay part are covered, so the relative size of perturbations describing a linear regime in a good approximation decreases. An increasing bandwidth for large feedbacks means the presence of high-frequency components in the time series, which require a higher resolution in time. While for the low feedback already the moderate step sizes reveal good results for the reconstructed sub-LE, the high bandwidth at high feedbacks needs to be covered by either a higher number of sampling points for the integral in the delay part of the local map or a reduction of the step size. With $K=1$ and $\theta_{1}=\delta t / 2$, i.e., a single sampling point for the delay term in the center of the integration interval, the approximation of the sub-LE improves by decreasing the step size $\delta t$, thus demonstrating the convergence for $\delta t \rightarrow 0$. A similar improvement of performance can be obtained by an increased delay integral sampling, where $K=2$, while keeping the step size fixed. This shows that the origin of the deviation from the correct sub-LE partially lies in the imperfect recovery of the delay integral. A combination of small step sizes and a proper reconstruction of the integral term yields further improvement if the available time series has enough data points and resolution.

The application of nonlinear time-series analysis methods to experimental optical systems with delay has successfully led to the identification and modeling of delay dynamics, as well as to the precise determination of the delay time in, e.g., a $\mathrm{CO}_{2}$-laser experiment with electro-optical feedback [16,30]. The algorithm described in this section is not restricted either to noiseless numerical time series. As an illustration, we compute the reconstructed sub-LE $\tilde{\lambda}_{0}$ for time series recorded from an optoelectronic experimental setup, which can be modeled using the Ikeda delay system [32,33]. This optoelectronic system is properly described by Eq. (11) after a time renormalization by a factor $t / T_{R}$, where $T_{R}$ is the response time of the system. Thus, it follows that the true sub-LE $\lambda_{0}=-1 / T_{R}$ in the experimental system.

The algorithm is applied to a time series with $N=400 k$ points, sampled at $10 \mathrm{~ns}$, using a splitting in $10 \%$ reference and $90 \%$ pool trajectory (see Appendix A). We use a step size of $\delta t=40 \mathrm{~ns}$ and $K=2$ support points for the delay term spaced by $\theta_{2}-\theta_{1}=20 \mathrm{~ns}$. The delay time of the system was estimated to be $\tau_{\exp }=20.79 \mu$ s using as the criterion the maximum of the autocorrelation function at the first delay echo, together with a small correction of $60 \mathrm{~ns}$. This correction corresponds to the minimum average fit error of the local linear map and accounts for the typical shift of the delay echoes in delay dynamical systems [21]. In Table I we present the extracted values of the sub-LE for varying experimental conditions, namely, different feedback values. Since the response time of the system under analysis is governed by a first-order low-pass filter with an experimentally measured response time $T_{R}=195 \pm 4 \mathrm{~ns}$, the expected sub-Lyapunov

TABLE I. Extracted values of the sub-LE for experimental time traces of an optoelectronic Ikeda delay system.

\begin{tabular}{lcc}
\hline \hline$\kappa$ & $\tilde{\lambda}_{0}\left(\mu \mathrm{s}^{-1}\right)$ & $\tilde{\lambda}_{0} / \lambda_{0}$ \\
\hline 2.8 & -5.04 & 0.982 \\
3.2 & -5.06 & 0.986 \\
3.6 & -5.05 & 0.984 \\
4 & -5.09 & 0.992 \\
4.4 & -5.08 & 0.990 \\
\hline \hline
\end{tabular}


exponent is $\lambda_{0}=-1 / T_{R}=(-5.13 \pm 0.1) \mu \mathrm{s}^{-1}$. As shown in Table I, the extracted values for $\tilde{\lambda}_{0}$ approximate the expected one for all feedback values. The relative error of the estimation $\left(\tilde{\lambda}_{0} / \lambda_{0}\right)$ is within a $2 \%$ margin, which is the size of the error of the experimentally determined value for the sub-LE.

\section{VECTOR SYSTEMS: FLOWS}

The most general case of a $d$-dimensional dynamical system with a fixed delay $\tau$ is given by

$$
\dot{\mathbf{x}}(t)=\mathbf{f}(\mathbf{x}(t), \mathbf{x}(t-\tau)),
$$

where the linearizations include the $d \times d$ Jacobians $U(t)=$ $D_{1} f(\mathbf{x}(t), \mathbf{x}(t-\tau))$ and $V(t)=D_{2} f(\mathbf{x}(t), \mathbf{x}(t-\tau))$. In order to calculate Lyapunov exponents the tangent system

$$
\dot{\delta} \mathbf{x}(t)=U(t) \cdot \delta \mathbf{x}(t)+V(t) \cdot \delta \mathbf{x}(t-\tau)
$$

needs to be recreated from the time series, which may be only a scalar recording $s(t)=g(\mathbf{x}(t))$. Before we address the issue of scalar recordings, we discuss the case that the entire trajectory $\mathbf{x}(t)$ has been recorded. Provided this, it is straightforward to recover the extension of the map (9) on a discretized time axis like in Sec. III,

$$
\delta \mathbf{x}_{n+1}=a_{n} \cdot \delta \mathbf{x}_{n}+\int_{t_{n}}^{t_{n+1}} d t b(t) \cdot \delta \mathbf{x}(t-\tau),
$$

where $a_{n}$ and $b(t)$ are $d \times d$ matrices. With a sampling in time as described in the previous section, nearest neighbors will be sought in a $(K+1) d$-dimensional space, which is why step size $\delta t$ and integral sampling $K$ need to be kept as small as possible in order to avoid large perturbation sizes.

\section{A. Application to vector data}

We apply the algorithm for the sub-LE to trajectories obtained by the Lang-Kobayashi model for semiconductor lasers with time-delayed feedback. It reads

$$
\begin{aligned}
& \dot{E}(t)=\frac{1+i \alpha}{2} G_{N} n(t) E(t)+\kappa E(t-\tau), \\
& \dot{n}(t)=(p-1) \gamma N_{\mathrm{sol}}-\left[\Gamma+G_{N} n(t)\right]|E(t)|^{2},
\end{aligned}
$$

with the parameters chosen like in Ref. [27] to be $G_{N}=2.142 \times 10^{-5} \mathrm{~ns}^{-1}, \Gamma=357 \mathrm{~ns}^{-1}, \gamma=0.909 \mathrm{~ns}^{-1}$, and $N_{\text {sol }}=1.707 \times 10^{8}$. The pump current of the laser enters as $p=I / I_{\text {th }}$, with $I_{\text {th }}$ being the threshold current of the solitary laser, and is set to $p=1.02$ only slightly above threshold. The feedback delay is set to $\tau=10 \mathrm{~ns}$, which corresponds to the long cavity regime, and the optical offset phase of the feedback is neglected. The feedback gain $\kappa$ serves as a free parameter to access different dynamical regimes, especially regimes of both strong and weak chaos. We integrate Eq. (13) using a Heun method with step size 0.1 ps. A transient part of $100 \tau$ is removed in order to obtain a stationary trajectory representative for the attractor of the system. Corresponding to the three-dimensional vector state of the system, there is a spectrum of three sub-LEs $\left(\lambda_{0, i}\right)$ for each trajectory. From the tangent linear system (12) we calculate the evolution operators $a_{n}$, to which we apply the canonical $Q R$-decomposition method [4,25]. The sub-LE spectrum as a function of the

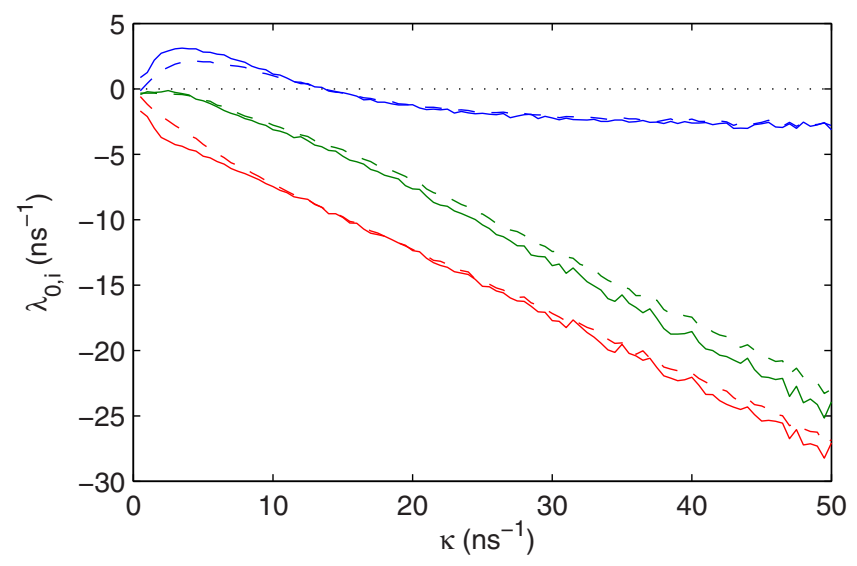

FIG. 3. (Color online) Spectrum of sub-LEs from the LangKobayashi equations as a function of the feedback strength $\kappa$ consisting of the maximum (blue), second (green), and minimum (red) exponents. Dashed lines indicate the true values calculated by a linearization. Solid lines denote the reconstructed values using nearest-neighbor reconstruction with a time step of $\delta t=10 \mathrm{ps}$, one support point in the center of the delay term integral $\left(\theta_{1}=5 \mathrm{ps}\right)$, and 200 nearest neighbors for the regression at each point. The full vector state of a trajectory of $1 \mu \mathrm{s}$ was used and another $1-\mu \mathrm{s}$ trajectory served as pool for nearest neighbors.

feedback $\kappa$ is shown in Fig. 3. While without feedback the subLEs are concentrated around zero, with increasing feedback there is a spread that is approximately proportional to the feedback rate. The lower exponents evolve as $\lambda_{0,2 / 3} \approx-\kappa / 2$ with a small and largely constant difference, whereas the maximum sub-LE shows a clearly different tendency with transitions from negative to positive and back to negative. These transitions of the maximum sub-LE correspond to the transitions of the dynamics from weak to strong chaos and back to weak chaos. Similar to the shape of the maximum sub-LE, the maximum LE of the system and its Kolmogorov-Sinai entropy also peak at intermediate feedback strengths $\kappa[14,34]$.

Using the entire three-dimensional vector state $\mathbf{x}(t)=$ $(\Re(E), \Im(E), n)^{\top}$ at time $t$ and the delayed counterparts around $t-\tau$ according to the sampling of the delay integral, we apply the algorithm as discussed before to the numerical time series. From the finite perturbations $\Delta \mathbf{x}_{j}\left(t_{n}\right)$ and $\Delta \mathbf{x}_{j}\left(t_{n}-\tau+\theta_{k}\right)$ the local linear map is reconstructed. We chose a small step size of $\delta t=10 \mathrm{ps}$ and a single sampling point for the integral located at its center, i.e., $K=1$ and $\theta_{1}=5 \mathrm{ps}$. With the approximated sequence of evolution operators $\tilde{a}_{n}$ our algorithm is able to reproduce the sub-LE spectrum with all its features. An almost exact agreement of the exponents for wide ranges of the feedback can be found. The largest deviations are located at small feedback rates, however, maintaining the shape of the curves and preserving the zero crossing of the maximum exponent at $\kappa=13 \mathrm{~ns}^{-1}$. The remaining error is related to nonlinear terms entering the local maps due to finite perturbation sizes as well as the imperfect sampling of the delay term integral.

\section{B. Application with scalar observables}

We want to apply the same algorithm for reconstruction of the local linear map and calculation of the sub-LE for the 
case that only a scalar observable of the vector state of the delay system is recorded. It is known that, in such a case, it is important to distinguish whether the delayed feedback is scalar or vectorial and whether the feedback variable is the one recorded or not in the case of scalar feedback [17]. For discrete maps, a proper embedding enables the recovery of the equivalent equations of motion in the embedding space rigorously for scalar feedback [17]. We follow a different approach aiming at continuous time systems with vectorial feedback.

In analogy to a typical experimental setup with a semiconductor laser subject to delayed optical feedback, we calculate the optical intensity $P(t)=|E(t)|^{2}$ from the same time series we used before. The direct application of the algorithm like in the case of the scalar time series from the Ikeda system in Sec. III would not make sense because the reconstructed map would suffer from false nearest neighbors originating from the projection in phase space. We thus follow the idea of phase space reconstruction using delay embedding. For clarity we refer in the following to the term lag to distinguish between the embedding time lags and the feedback delay of the system. In the case of the drive-response setup, Pyragas showed that the embedding method is useful, as soon as the embedding dimension sufficiently covers all degrees of freedom of the entire system consisting of the drive and response [25]. Although it appears reasonable to consider $P(t-\tau)$ as a drive variable and $P(t)$ as an observable of the response system, we will not be able to meet the necessary conditions in our case. This is due to the fact that the drive in the delay system originates from a driven system, in the sense that the state at $t-2 \tau$ acts on the state at $t-\tau$ and analogously for further multiples of the delay. Apparently the entire complexity of the high-dimensional delay dynamics enters here, so the reduction to the simple drive-response scheme may fail. Reference [35] comprises a study on Taken's embedding theorem for driven systems. Here we consider the incompleteness of the vector-state reconstruction as an additional source of error and apply the algorithm directly in order to obtain an estimate for the effect of this error. We apply the embedding part of the Pyragas algorithm as for the case of a drive-response relationship with the embedding dimension being the dimension of the node of the delay system. Further, we also use the method of setting embedding lags equal to the step $\delta t$ of the map, which by construction reduces the number of fit parameters. The resulting local linear map for the three-dimensional embedding of the simulated laser intensity on a grid $t_{n}=n \delta t$ reads

$$
\Delta P_{j, n+1}=\tilde{\mathbf{a}}_{n} \cdot \Delta \mathbf{P}_{j, n}+\tilde{\mathbf{b}}_{n} \cdot \Delta \mathbf{P}_{j, n, \tau}+\epsilon_{j, n} .
$$

It forms the reconstructed neighborhood of $P_{n+1}=P\left(t_{n+1}\right)$ as well as the embedded phase space vectors $\mathbf{P}_{n}=\left(P\left(t_{n}\right), P\left(t_{n-1}\right)\right.$, $\left.P\left(t_{n-2}\right)\right)^{\top}$ and $\mathbf{P}_{n, \tau}=\left(P\left(t_{n}-\tau\right), P\left(t_{n-1}-\tau\right), P\left(t_{n-2}-\tau\right)\right)^{\top}$. The fit coefficients are linear forms $\tilde{\mathbf{a}}_{n}=\left(\tilde{a}_{n, 1}, \tilde{a}_{n, 2}, \tilde{a}_{n, 3}\right)$ and $\tilde{\mathbf{b}}_{n}=\left(\tilde{b}_{n, 1}, \tilde{b}_{n, 2}, \tilde{b}_{n, 3}\right)$. Nearest neighbors are sought in the joint space of $\left(\mathbf{P}_{n}, \mathbf{P}_{n, \tau}\right)$ to construct the perturbation vectors. In analogy to the conditional Lyapunov exponents that are being calculated from the $\tilde{\mathbf{a}}_{n}$ in the case of drive-response schemes, we calculate the sub-LE spectrum for the delay system. The results for the spectrum of the Lang-Kobayashi trajectories are

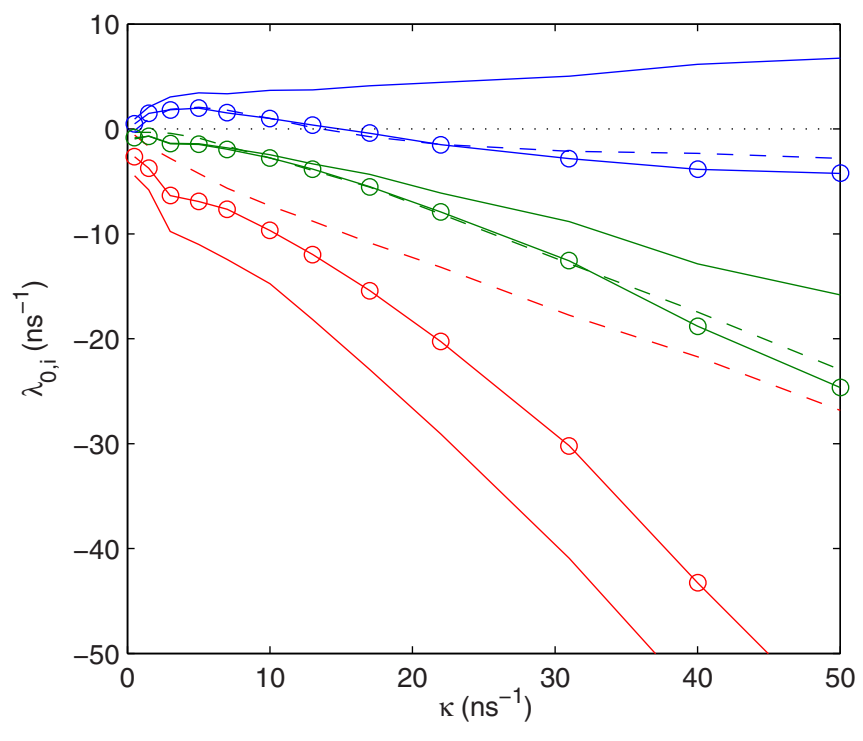

FIG. 4. (Color online) Spectrum of sub-LEs from the LangKobayashi equations as a function of the feedback strength $\kappa$ consisting of the maximum (blue), second (green), and third (red) exponents. Dashed lines indicate the true values calculated by a linearization. Solid lines (both with and without markers) denote the reconstructed values using nearest-neighbor reconstruction with a time step of $\delta t=10 \mathrm{ps}$. The total length of the time series was $20 \mu$ s or $N=2 \times 10^{6}$ points with splitting in $5 \%$ reference and 95\% pool; see Appendix A. Only the intensity of the simulated semiconductor laser was used as a scalar observable of the trajectory underlying the calculation of the spectrum. Solid lines without markers denote the result obtained by simple embedding using three support points at $\left(t_{n}, t_{n-1}, t_{n-2}\right)$ and three support points for the first delay at $\left(t_{n}-\tau, t_{n-1}-\tau, t_{n-2}-\tau\right)$. Solid lines with markers denote the result of the extended embedding using also the past state two delay times before at $\left(t_{n}-2 \tau, t_{n-1}-2 \tau, t_{n-2}-2 \tau\right)$.

shown as solid lines (without markers) in Fig. 4. We observe that general features of the spectrum like the spread with increasing feedback rate and the approximate location of the exponents are already reproduced. Still, the results are clearly not as good as those presented in Fig. 3, indicating the impact of the error from embedding, although except for the embedding procedure there are no major procedural changes compared to the reconstruction using the vector states in Sec. IV A.

\section{Extended embedding}

We propose a method to overcome the limitations of the phase space reconstruction using extended lag embedding. The idea is based on the iterative drive argument presented above. The linear map as given by Eq. (14) is incomplete in the sense that also information from further multiples of the delay time causally acts on $P_{n+1}$. In particular, the state of the drive, i.e., the delayed vector state $\mathbf{x}(t-\tau)$ of the node, can be considered as insufficiently reconstructed by the vector $\mathbf{P}_{n, \tau}$. Thus we extend the drive state by the information available another delay time earlier. The extended vector $\mathbf{P}_{n, \tau}=\left(P\left(t_{n}-\tau\right), P\left(t_{n-1}-\tau\right), P\left(t_{n-2}-\tau\right)\right.$, $\left.P\left(t_{n}-2 \tau\right), P\left(t_{n-1}-2 \tau\right), P\left(t_{n-2}-2 \tau\right)\right)^{\top}$ is incorporating information from a previous delay interval. Accordingly, the 
linear form for the delay term is extended as $\tilde{\mathbf{b}}_{n}=$ $\left(\tilde{b}_{n, 1}, \ldots, \tilde{b}_{n, 6}\right)$. The increased dimensionality is a clear drawback for the performance of the algorithm because nearest neighbors will be sought in a high-dimensional space and will be more distant than in the case of lower dimensions. Still, with the presented number of dimensions and length of the time series as described above, one obtains reasonable performance on standard computers. Except for the extended embedding of the delay term, we leave the algorithm as before. The outcome of the extended technique is shown as solid lines with markers in Fig. 4 in comparison with the simple lag embedding of the delay term as well as with the true exponents. Underlying time series and algorithm parameters are the same. Although the increased dimensionality also leads to increased nearest-neighbor distances because the total length of the time series is kept constant, we recognize a clear improvement of the reconstructed sub-LE spectrum. The leading exponent is reconstructed almost perfectly, in particular the zero crossing is reproduced. The reconstruction of the middle exponent can also be regarded as excellent, while the lower exponent still shows a big deviation from its true value. However, the improvement compared to the simple lag embedding is already visible also in the lowest exponent. Among other possible extensions of the algorithm for scalar observables, we regard the incorporation of multiple delays as the method yielding the most significant improvement.

\section{DISCUSSION AND OUTLOOK}

We have developed and characterized a robust algorithm to compute the sub-Lyapunov exponent from time series of delay systems in the chaotic regime. The algorithm is based on the reconstruction of the local linearized equations of motion, as has been laid out by Bünner and co-workers [15,17] and Hegger and co-workers [16,31]. This representation of a delay system directly leads to a drive-response description, in which the sub-Lyapunov exponent is a property of the response system. Since our aim is the analysis of continuous vector-state systems, we construct our method in three steps.

First, we use the example of the logistic map with delay to introduce the reduction to a local map and we define metric distances in the space formed by the instantaneous and delayed variables for the nearest-neighbor search. Second, we present the scalar, continuous, Ikeda delay system to discuss the influence of a discretization time step and the approximation of the functional integral part. Third, we study vector systems and show that the algorithm can extract the true values of the sub-LE when the full information of the system is known. Finally, we tackle the challenge of having access to a single scalar observable of the vector system and show that the algorithm, in this case, requires an extended lag embedding. For all the numerical configurations covered in this paper, the algorithm is remarkably precise and recovers the true value of the sub-LE. In addition, we show by example that this algorithm is robust to noise and can extract the sub-LE from experimental time series.

Our method faces common trade-offs because the calculation of Lyapunov exponents is formally based on limits, which in practice cannot be met simultaneously. For instance, the finite size of perturbations is a dominant source of error because nonlinear terms of the local vector field are involved. This issue might be addressed by extrapolation techniques, which could serve also for taking the limit of a small step size. There is also potential for extensions and adaptations as well as for estimations of the error when applying embedding to scalar recordings of vector data. Measuring multivariate experimental time traces could clearly support our method here and for the dynamics of semiconductor lasers it has recently been shown to be possible to obtain the required information [36].

The presented perspective, in which the node of the delay system is considered as a response system, allows for accessing intrinsic dynamical properties, which otherwise would be practically impossible. With the applicability to real world data the method is very promising for extracting relevant information from physical systems. The recovery of the subLE will also be helpful in a context where the transformation by the nonlinear node needs to be tailored for an application. Beyond the results presented here, where an entire trajectory is only described by a single exponent, the presented algorithm will also allow for the study of finite-time Lyapunov exponents. In multiscale systems a phase space resolved version of the sub-LE can provide valuable information about the local dynamics. In conclusion, given the relevance of the sub-LE for analysis as well as application of delay systems and given the difficulty to properly analyze such high-dimensional systems in general, this paper paves the way to a better characterization of delay systems from time series.

\section{ACKNOWLEDGMENTS}

This work was supported by a fellowship within the Postdoc-Programme of the German Academic Exchange Service (DAAD), FEDER and MINECO (Spain) under Project No. TEC2012-36335 (TRIPHOP) and Comunitat Autonoma de les Illes Balears via Grups Competitius.

\section{APPENDIX A: TECHNICAL IMPLEMENTATION}

\section{Splitting time series}

Practically, in order to avoid nearest neighbors being close in time to a reference point, we implemented an asymmetric procedure consisting of a reference time trace and another equivalent time trace acting as a pool from which nearest neighbors are chosen. The reason for this choice lies in the amount of computation. While for the example of a typical Lang-Kobayashi trajectory a reference time series of $1 \mu \mathrm{s}$ is sufficient in terms of the convergence of the finite-time Lyapunov exponent to its limit value, one may need a much longer trajectory serving as a pool for nearest neighbors of the reference points in order to obtain sufficiently small nearest-neighbor distances. We particularly make use of this asymmetry in Sec. IV C, in which we adapt the algorithm to work with scalar observables of the vector state.

\section{Nearest-neighbor search}

The space of the state variables of the nonlinear node, whether they are the original ones or substitutes from lag embedding, is not a priori a metric space. The choice of 
the norm defining distances between two points is arbitrary and theoretically does not affect the calculation of Lyapunov exponents, when working with infinitesimal perturbations and taking the limit of infinite time for averaging. In the context of our study, where the limit conditions are not completely met, the choice of a proper norm is relevant. We apply the Euclidean norm after guaranteeing that the phase space variables are sufficiently uncorrelated. If this is the case, the result hardly depends on the applied norm. We confirmed this using instead the maximum norm and also a mixed norm, which first takes the Euclidean distance in the instantaneous vector and the delayed vector separately and then measures the maximum of both. For the scalar observable of the vector state, in which we linked the embedding lag to the step size $\delta t$ given by the sampling, we found that one obtains the best results in terms of lowest fit error and closest approximation of the sub-LE if the step size is taken to be small compared to the time of the first zero crossing of the autocorrelation function. This choice is unusual in terms of the typical embedding lags because it naturally leads to a prolate attractor projection. We unfold this set of points using a Gram-Schmidt orthogonalization separately on the instantaneous vector and the delayed vector before applying the standard norm.

\section{APPENDIX B: ROBUSTNESS}

The minimal number of nearest neighbors to construct small perturbations around a reference point is given by the number of coefficients. With the linear regression method the minimal number is higher, typically by a factor 2 , because of singular matrices appearing frequently during the calculation. For our calculations we use a much higher number of nearest neighbors than technically necessary. This method has three advantages if sufficient data are available. First, the fit with $J \gg J_{\min }$ introduces a certain robustness with respect to additive noise. Second, the results become insensitive to second-order nonlinear terms that may play a role for finite perturbations. Finally, a dynamical error occurs even in a noise-free system when approximating the integral part of the delay term in the continuous case. We demonstrate these issues by means of application of our algorithm to time series from the logistic map with delay and the continuous delay Ikeda system like in Secs. II and III.

\section{Additive noise}

We add measurement noise to the trajectories of the delayed logistic map such that $x_{n} \rightarrow x_{n}^{\prime}=x_{n}+r \xi_{n}$ with $\xi_{n}$ being normally distributed random numbers with unit variance. The reconstruction algorithm is applied to the time series $x_{n}^{\prime}$ to calculate the sub-LE from the obtained coefficients $\tilde{a}_{n}$. The standard deviation $r$ of the noise term is compared to the average size of the perturbations ${\overline{\Delta x^{\prime}}}^{2}=\left\langle\Delta x_{j, n}^{\prime 2}\right\rangle$ from nearest neighbors yielding the effective noise strength $r_{\text {eff }}=r / \overline{\Delta x^{\prime}}$. Figure 5 shows a comparison of the obtained exponents with three clearly distinct numbers of nearest neighbors, where the length of the pool trajectory has been adapted proportionally such that the average size of perturbations is the same and the results are comparable. The noise term leads to overor underestimation of the sub-LE. For increasing $J$, the

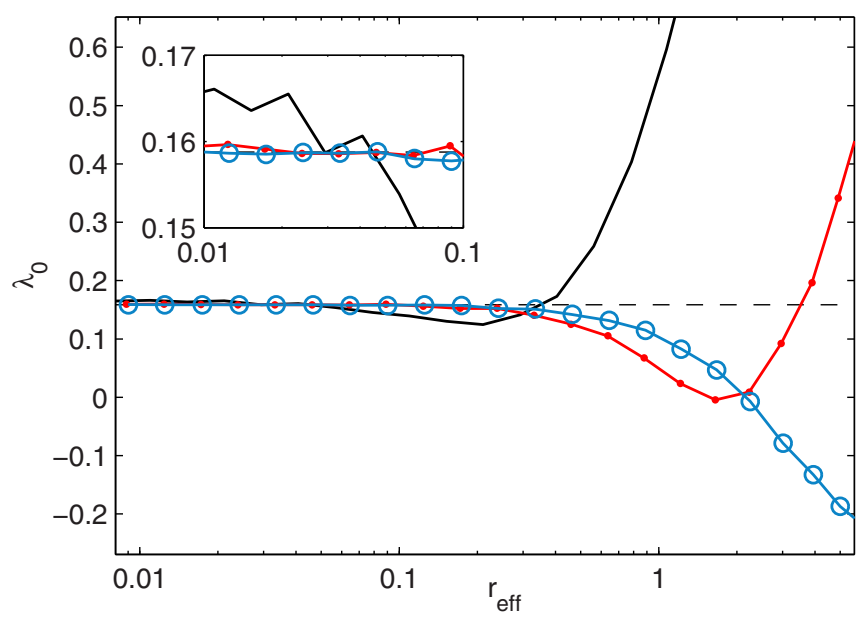

FIG. 5. (Color online) Reconstruction of the sub-LE of the delayed logistic map at feedback $\kappa=0.25$ with additive Gaussian measurement noise. The true exponent $\lambda_{0}=0.1588$ is indicated by the dashed horizontal line. The horizontal axis is the effective noise strength $r_{\text {eff }}$. The reconstructed exponent is shown for $J=4$ (black), $J=40$ (red with dots), and $J=400$ (blue with circles), with pool sizes $2 \times 10^{3}, 2 \times 10^{4}$, and $2 \times 10^{5}$, respectively.

reconstructed $\tilde{\lambda}_{0}$ converges to the true $\lambda_{0}$ if $r<\overline{\Delta x^{\prime}}$. We further note a small offset of the exponent for small $J$ in the limit of low noise, which can be explained by nonlinear terms affecting the finite perturbations and is compensated by large $J$ as well.

\section{Dynamical error}

For the time series of the continuous Ikeda delay system, there are two sources of error in the reconstructed local linear map, namely, nonlinearities affecting finite perturbations and the finite sampling of the delay integral term. One may distinguish the contributions to the approximation error in the sub-LE by varying parameters of the algorithm such that either one or the other source can be excluded from the effect. We demonstrate here that the fit using a large number of nearest neighbors can partially compensate for such an error. At an intermediate feedback rate of $\kappa=10$ we apply the reconstruction algorithm to the time series using different

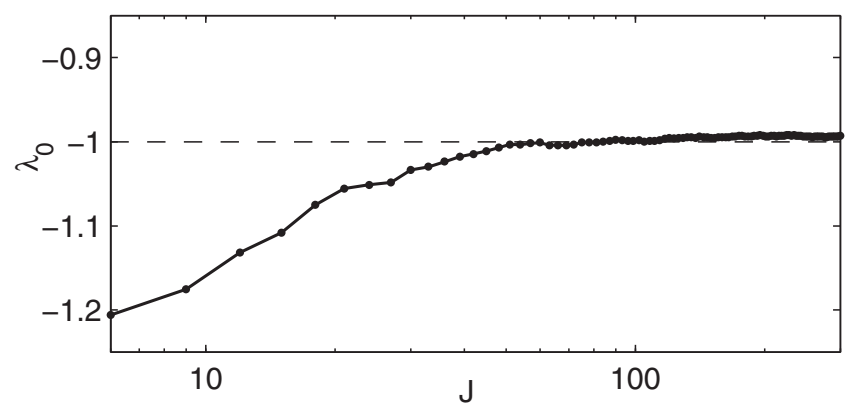

FIG. 6. Convergence of the reconstructed sub-LE of the numerical Ikeda system (solid line with markers) to the true sub-LE $\lambda_{0}=-1$ (dashed line) with increasing number of nearest neighbors $J$ at feedback strength $\kappa=10$. 
numbers $J$ of nearest neighbors. The length of the pool time series is adapted proportionally to keep the average size of the perturbations and hence the average error of a single perturbation constant. Figure 6 shows the convergence of the reconstructed $\tilde{\lambda}_{0}$ to the true $\lambda_{0}=-1$ for increasing $J$. Around $J=100$ the convergence is on the order of the remaining error, which originates from higher-order nonlinearities and the finite reference trajectory.
[1] A. Wolf, J. B. Swift, H. L. Swinney, and J. A. Vastano, Phys. D: Non. Phenom. 16, 285 (1985).

[2] M. Sano and Y. Sawada, Phys. Rev. Lett. 55, 1082 (1985).

[3] J. Eckmann and D. Ruelle, Rev. Mod. Phys. 57, 617 (1985).

[4] J.-P. Eckmann, S.-O. Kamphorst, D. Ruelle, and S. Ciliberto, Phys. Rev. A 34, 4971 (1986).

[5] H. Kantz and T. Schreiber, Nonlinear Time Series Analysis (Cambridge University Press, Cambridge, 1997).

[6] F. Takens, in Dynamical Systems and Turbulence, Warwick 1980, edited by D. Rand and L.-S. Young, Lecture Notes in Mathematics Vol. 898 (Springer, Berlin, 1981), pp. 366-381.

[7] H. S. Greenside, A. Wolf, J. Swift, and T. Pignataro, Phys. Rev. A 25, 3453 (1982).

[8] T. Erneux, Applied Delay Differential Equations (Springer, Berlin, 2009).

[9] M. Mackey and L. Glass, Science 197, 287 (1977).

[10] J. D. Farmer, Physica D 4, 366 (1982).

[11] K. Ikeda and K. Matsumoto, Physica D 29, 223 (1987).

[12] V. Ahlers, U. Parlitz, and W. Lauterborn, Phys. Rev. E 58, 7208 (1998).

[13] J.-P. Goedgebuer, L. Larger, and H. Porte, Phys. Rev. Lett. 80, 2249 (1998).

[14] R. Vicente, J. Dauden, P. Colet, and R. Toral, IEEE J. Quantum Electron. 41, 541 (2005).

[15] M. J. Bünner, M. Popp, T. Meyer, A. Kittel, and J. Parisi, Phys. Rev. E 54, R3082 (1996).

[16] R. Hegger, M. J. Bünner, H. Kantz, and A. Giaquinta, Phys. Rev. Lett. 81, 558 (1998).

[17] M. J. Bünner, M. Ciofini, A. Giaquinta, R. Hegger, H. Kantz, R. Meucci, and A. Politi, Eur. Phys. J. D 10, 165 (2000).

[18] F. T. Arecchi, G. Giacomelli, A. Lapucci, and R. Meucci, Phys. Rev. A 45, R4225 (1992).
[19] M. C. Soriano, J. García-Ojalvo, C. R. Mirasso, and I. Fischer, Rev. Mod. Phys. 85, 421 (2013).

[20] L. Larger, J.-P. Goedgebuer, and V. Udaltsov, C. R. Phys. 5, 669 (2004).

[21] X. Porte, O. D’Huys, T. Jüngling, D. Brunner, M. C. Soriano, and I. Fischer, Phys. Rev. E 90, 052911 (2014).

[22] B. Tromborg and J. Mork, IEEE J. Quantum Electron. 26, 642 (1990).

[23] A.-S. Landsman and I.-B. Schwartz, Phys. Rev. E 75, 026201 (2007).

[24] L. M. Pecora and T. L. Carroll, Phys. Rev. Lett. 64, 821 (1990).

[25] K. Pyragas, Phys. Rev. E 56, 5183 (1997).

[26] S. Heiligenthal, T. Dahms, S. Yanchuk, T. Jüngling, V. Flunkert, I. Kanter, E. Schöll, and W. Kinzel, Phys. Rev. Lett. 107, 234102 (2011).

[27] S. Heiligenthal, T. Jüngling, O. D’Huys, D. A. Arroyo-Almanza, M. C. Soriano, I. Fischer, I. Kanter, and W. Kinzel, Phys. Rev. E 88, 012902 (2013).

[28] X. Porte, M. C. Soriano, and I. Fischer, Phys. Rev. A 89, 023822 (2014).

[29] N. Oliver, T. Jüngling, and I. Fischer, Phys. Rev. Lett. 114, 123902 (2015).

[30] M. J. Bünner, M. Ciofini, A. Giaquinta, R. Hegger, H. Kantz, R. Meucci, and A. Politi, Eur. Phys. J. D 10, 177 (2000).

[31] R. Hegger, Phys. Rev. E 60, 1563 (1999).

[32] L. Larger, J. P. Goedgebuer, and J. M. Merolla, IEEE J. Quantum Electron. 34, 594 (1998).

[33] M. C. Soriano, L. Zunino, L. Larger, I. Fischer, and C. R. Mirasso, Opt. Lett. 36, 2212 (2011).

[34] L. Zunino, O. Rosso, and M. C. Soriano, IEEE J. Sel. Top. Quantum Electron. 17, 1250 (2011).

[35] J. Stark, D. Broomhead, M. Davies, and J. Huke, Nonlinear Anal. Theor. 30, 5303 (1997).

[36] D. Brunner, M. Soriano, X. Porte, and I. Fischer (unpublished). 